more from the same training. The conclusion seems to be, the better the student, the more he is able to profit by training, and, conversely, the greater the training, the greater the differences between those of higher and lower ability.

UNIVERSITY OF TEXAS.

$$
\text { E. E. RaLL. }
$$

Normal Performance in the Tapping Test. F. L. Whlls. Amer. J. of Psychol., 1908, XIX., 437-483.

This is an attempt to further standardize an important psychological measure, that of the maximum rate of repeated voluntary movements. The taps were made upon a telegraph key which was in circuit with a magnet recording upon smoked paper.

The subject began at a given signal and tapped at a maximum rate for thirty seconds; after a rest of two and one-half minutes another series was taken; and so on until a set of five such series had been obtained from each hand. The experiment was evaluated by counting the number of times the key was struck during each of the six five-second intervals of a series of thirty seconds.

The extremes of individual variation for ten unpracticed subjects were in initial rate approximately $2: 1$, in the gross rates for thirty seconds about $3: 2$; but it does not appear that this difference is related to general quickness, and beyond efficiency of coördination it is not known upon what normal condition it does depend.

The high gross rate in tapping does not, as has been pointed out, involve superiority in other aspects of motor speed.

Using Woodworth's ' index of right-handedness,' based on the ratioof the efficiency of the left hand to that of the right, the criterion in this case is the average number of taps, executed during five series of 30 seconds each. In the average, left hand does relatively better when it precedes than when it follows the right, which would be the case if the work with one hand had a fatiguing effect upon the speed of the other, but in the individual cases this is seen to be the product of certain coarse deviations in either direction, so that no general conclusion should be drawn. This index of right-handedness here varies between .81 and .94 , and shows distinct points of individual difference.

In the right hand it is quite noticeable that the first two series are generally the poorest and that there is a well marked tendency for the later series to be faster than the earlier. To this phenomenon he applies the name of 'interserial warming up.' In the left hand it is by no means so evident that such a process exists. After the first few seconds, the rate 
in the individual series progressively decreases, having, on the average, some six-sevenths as much speed during the last five seconds as in the first five, the curve following the form usual to fatigue curves. For the more thoroughly studied individuals the practice curve is everywhere gradual in ascent, not most rapid at the beginning, the opposite of what we usually expect. The practice curve of the left hand does not ascend more rapidly than the right. And variations from a parallel course seem to be due to the fact that each hand does relatively better if tested after rather than before the other. This suggests that the effects are of a general nature. Practice increases the effect of the warming-up tendency, which therefore shows itself much more in the later experiments than in the earlier. The intermissions of two weeks or less have no unfavorable effect on the practice gain beyond at first increasing fatigue sensations.

There is only a slight tendency to positive correlation in the fatigability of the different hands. The right hand is ordinarily more immune to fatigue than the left; but there are individual exceptions. Practice tends to affect the average 'index of fatigue' of the left hand more favorably than that of the right. Initial rate and fatigability are negatively correlated, a fast initial rate being usually accompanied by a high fatigue loss. Fatigue tends in all respects to decrease variability. No consistent effect on variability due specifically to 'warming-up' or practice can be traced. The mean variation in the right hand tends to be larger than that for the left hand.

The subjective condition as estimated by an individual practiced in introspection bore no traceable relationship to the gross rate; it seemed, however, that the susceptibility to fatigue was greater when the grade assigned was good than when it was poor. Subjective condition and initial rate are more closely correlated.

Practice decreases the ratio of the period during which the key is held down to that during which it is released.

UNIVERSITY OF MICHIGAN.

H. W. Carpenter.

\section{FATIGUE AND DRUGS.}

The Influence of Alcohol and Other Drugs on Fatigue. W. H. R. Rivers. The Croonian Lectures, Igo6. London, Arnold, igo8. Pp. viii +136 .

Although primarily undertaken to demonstrate the pharmacological action of drugs on the neuromuscular apparatus, the present work of Rivers is of considerable psychological interest because it shows con- 